\title{
Age-gender normal values of native and post- contrast myocardial T1 relaxation times (lambda) on 1.5T and 3T using MOLLI: a multicenter, single vendor cardiovascular magnetic resonance study
}

\author{
Darius Dabir ${ }^{1}$, Nicholas Child ${ }^{1}$, Ashwin Kalra', Islam Z Mahmoud ${ }^{1}$, Toby Rogers ${ }^{1}$, Rolf Gebker ${ }^{4}$, Ananth Kidambi ${ }^{3}$, \\ Sven Plein ${ }^{3}$, Andrew Jabbour ${ }^{5}$, David M Higgins², Bernhard Schnackenburg ${ }^{2}$, Tobias Schaeffter ${ }^{1}$, Eike Nagel ${ }^{1}$, \\ Valentina Puntmann ${ }^{1 *}$
}

From 17th Annual SCMR Scientific Sessions

New Orleans, LA, USA. 16-19 January 2014

\section{Background}

Myocardial T1 mapping is emerging as a promising means to non-invasively discriminate between normal and diseased myocardium. We have shown that $\mathrm{T} 1$ measurements perfromed conservatively within the septal myocardium are reproducible and accurate with excellent discriminatory ability between normal and abnormal myocardium. Using this approach we aimed to determine age and gender related normal values at clinically used field strengths, 1.5 Tesla (T) and 3T, in a multi centre and single vendor study.

\section{Methods}

We recruited normotensive subjects with no cardiovascular risk factors, low pretest probability of cardiovascular or systemic disease, taking no regular medication and subsequently, a normal CMR study (normal LV volumes, mass and no LGE), underwent native and post-contrast $\mathrm{T} 1$ imaging with modified look-locker inversion recovery (MOLLI; 3,3,5) either at $1.5 \mathrm{~T}$ or $3 \mathrm{~T}$ (Achieva, Philips Healthcare, Best, The Netherlands). Parameters for native and post-contrast MOLLI were identical (FOV $320 \times 320$; TR/TE/flip-angle: $3.3 \mathrm{~ms} / 1.57$ $\mathrm{ms} / 50^{\circ}$, interpolated voxel size $0.9 \times 0.9 \times 8 \mathrm{~mm}$, phase encoding steps $\mathrm{n}=166$, HR adapted trigger delay, with 11 (3-3-5) phase sampling arrangements. Adiabatic prepulse was used to achieve complete inversion. Septal ROIs were automatically propagated across all eleven images in the MOLLI sequence with a prior image- coregistration step for motion-correction (figure).

\section{Results}

Two-hundred and thirty subjects were enrolled (age (years) median:43 (17-75), male: $\mathrm{n}=118,51 \%, 1.5 \mathrm{~T}$ vs. $3 \mathrm{~T}$ : $\mathrm{n}=114$ vs. 116 . Mean (min-max; SD) T1 values (msec) per field strength were: native T1: 954 (896-997; 23) and

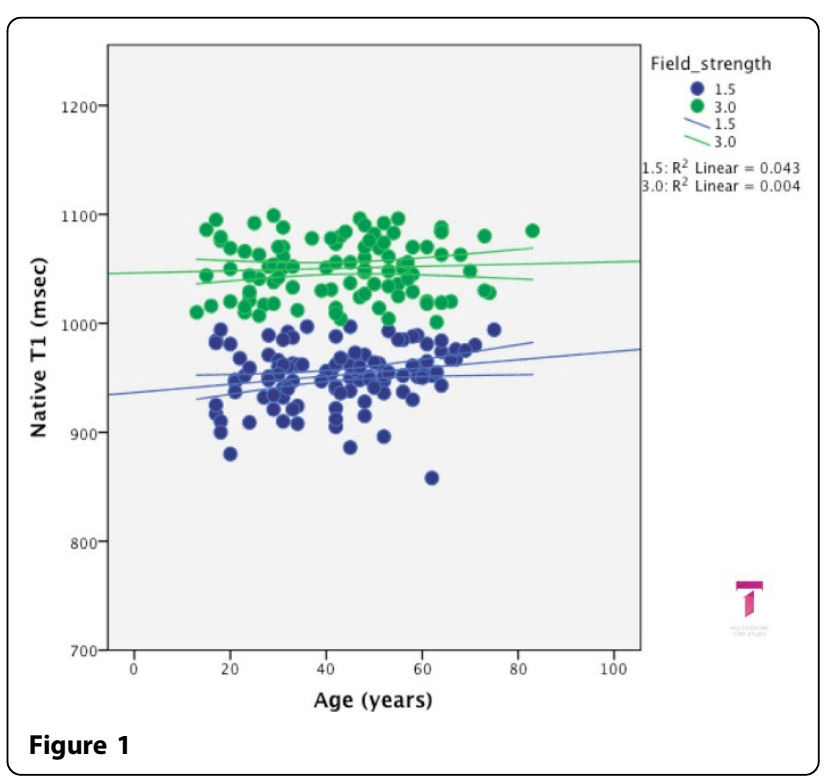

${ }^{1}$ King's College London, London, UK

Full list of author information is available at the end of the article 


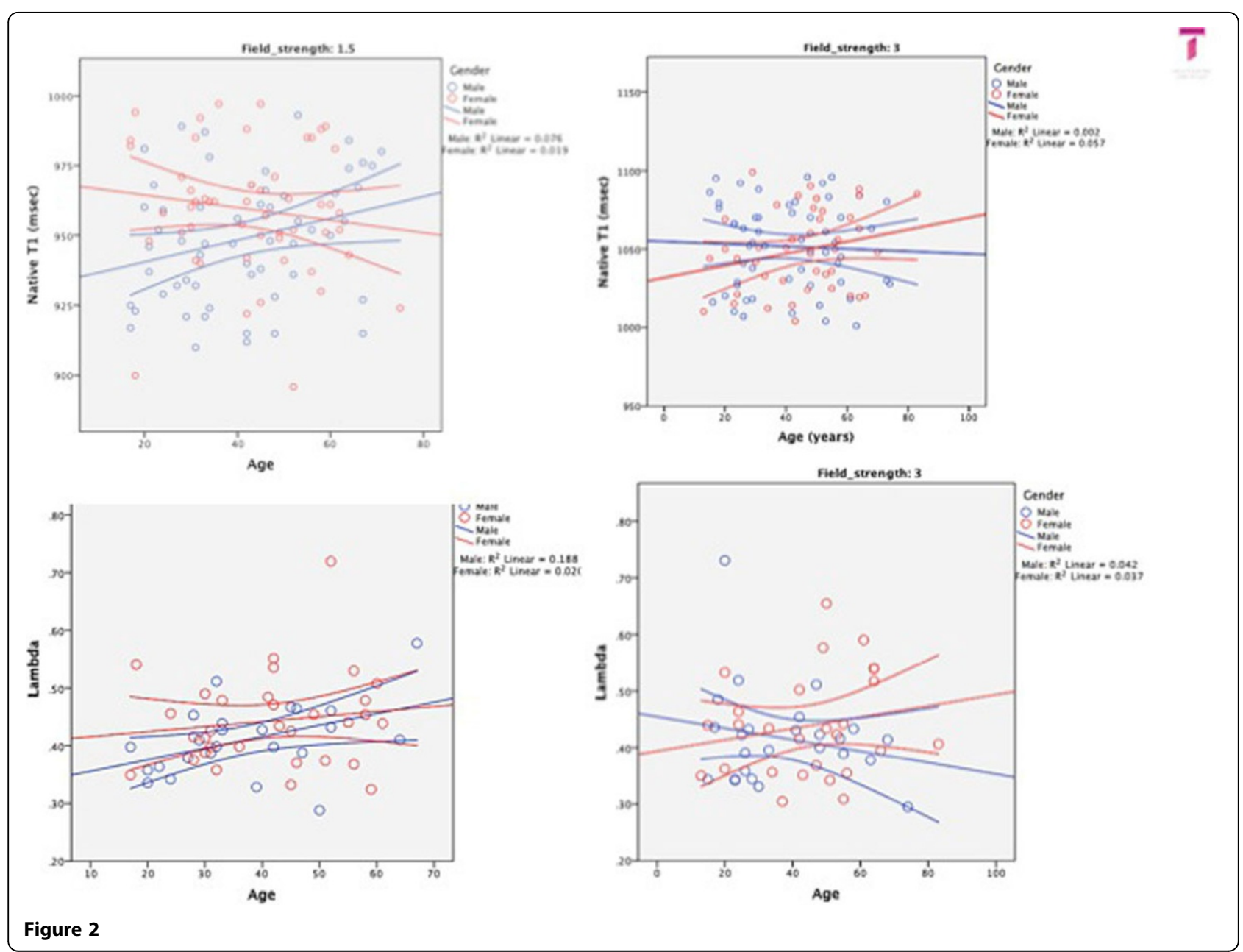

1052 (1001-1099; 27); post contrast T1: 400 (303-546; 59), lambda (\%): 43 (29-72;9). There were no differences for gender or association with age for any of the $\mathrm{T} 1$ values or derivatives (Figure 1, 2).

\section{Conclusions}

We report normal values for $\mathrm{T} 1$ values and derivatives, based on 3'3'5MOLLI sequence and using conservative septal sampling approach approach. We demonstrate no age or gender related differences at either field strengths.

\section{Funding}

We would like to acknowledge Department of Health via the National Institute for Health Research (NIHR) comprehensive Biomedical Research Centre award to Guy's \& St Thomas' NHS Foundation Trust in partnership with King's College London and King's College Hospital National Health Service Foundation Trust.

\section{Authors' details}

${ }^{1}$ King's College London, London, UK. P2Philips Healthcare, Guilford, UK. ${ }^{3}$ Leeds University, Leeds, UK. ${ }^{4}$ German Heart Institute Berlin, Berlin, Germany. ${ }^{5} \mathrm{St}$ Vincent University, Sydney, New South Wales, Australia.

Published: 16 January 2014

doi:10.1186/1532-429X-16-S1-P23

Cite this article as: Dabir et al:: Age-gender normal values of native and post-contrast myocardial T1 relaxation times (lambda) on 1.5T and 3T using MOLLI: a multicenter, single vendor cardiovascular magnetic resonance study. Journal of Cardiovascular Magnetic Resonance 201416 (Suppl 1):P23. 